\title{
Experimental study on the effect of graphene on the thermal conductivity of natural rubber latex
}

\author{
Lin $\mathrm{Li}^{1}$, Wen Huang ${ }^{1}$, Yingchao $\mathrm{Ma}^{1}$ Chaoping $\mathrm{Hou}^{1,2 *}$ \\ ${ }^{1}$ Dujiangyan Yunminongqing Science and Technology Co. LTD., Dujiangyan, Chengdu, Sichuan, China. \\ ${ }^{2}$ College of Architecture and Urban-Rural Planning, Sichuan Agricultural University., Dujiangyan, Chengdu, Sichuan, China.
}

\begin{abstract}
Graphene is a new type of carbon material with excellent performance. It not only has good electrical, mechanical and thermal properties, but also can be used as a filler of natural latex materials to optimize the thermal conductivity of natural latex materials. Therefore, graphene is widely used in aviation, automobile, machinery and other fields. In order to study the influence of the coordination ratio of graphene and rubber materials on the thermal conductivity, the natural rubber latex was first diluted and dissolved with volatile organic solvent toluene; then $0 \mathrm{~g}, 0.5 \mathrm{~g}, 1 \mathrm{~g}, 1.5 \mathrm{~g}, 2 \mathrm{~g}$, and graphene were respectively mixed into $20 \mathrm{~g}$ natural rubber latex to prepare 6 thin samples (type I, type II, type III, type IV, type V, type VI). Finally, the thermal conductivity of 6 samples was measured by fla laser thermal conductivity instrument. The results showed that with the increase of graphene dosage, the thermal conductivity of natural rubber latex sheet increased significantly; when the ratio of graphene dosage to natural rubber latex was about 1:10, the rate of improving thermal conductivity of natural rubber latex decreased.
\end{abstract}

\section{Introduction}

Due to its excellent physical and chemical properties, graphene has great application prospects in polymer nanocomposites [1]. In practical application, natural rubber latex can improve not only mechanical strength and wear resistance of materials, but also the conductivity and thermal conductivity of natural latex composites, as well as the gas barrier property of [2-4]. Zhou Wang filled graphene into the epoxy resin, so he got the conclusion that graphene can improve the strength and flame retardancy of the epoxy resin [5]; everelt C Salas compounded graphene with cytochrome, so that graphene can be used as a probe to detect the density of Escherichia coli in water [6]; Zhong-shuai Wu doped graphene with nitrogen and boron to modify graphene from the molecular structure to prepare graphene-based electrodes [7]; Jun Yong Sun modified graphene with acoflavin to obtain graphene/acoflavin composite [8]. Longun $J$ et al. [9] dispersed the nanometer graphene sheets uniformly in the matrix of poly (phthalimide) through in-situ condensation polymerization. It is found that the modulus of flat area is obviously higher than that of glass state. Xu Y F et al. [10] prepared a new type of graphene composite by in-situ polymerization, which has excellent conductivity, good flexibility and good thermal stability. The conductivity of $10 \mathrm{~mm}$ film is $0.2 \mathrm{~s} / \mathrm{m}$, and the light transmittance exceeds $80 \%$.This transparent, flexible, electrically conductive graphene composite has applications in a variety of optoelectronic devices.
In conclusion, most of the domestic and foreign scholars have studied graphene. The results show that the composite of graphene and natural latex can improve the thermal conductivity of the material. However, the effect of the ratio of graphene to natural latex on the thermal conductivity is rarely studied. In this experiment, six kinds of samples were prepared according to the different proportion of graphene and natural latex. The thermal conductivity of six samples was measured by fla laser thermal conductivity meter, and the influence of different matching ratio on the thermal conductivity of graphene and natural latex composite was studied.

\section{Materials and methods}

\section{1 experimental materials}

Natural rubber latex is a natural latex liquid material that is not directly cured after natural rubber is cut from rubber tree. It has many uses in the processing and preparation of rubber products [11]. Natural rubber latex comes from the gum of rubber trees and other plants, so it is usually not pure rubber. According to the national standard gb8299-2008, the rubber content of natural rubber latex is $65.2 \%$ [12]. The viscosity of pure natural rubber latex is relatively large by mixing all kinds of additives in rubber formula, so the natural rubber latex is usually diluted by volatile organic solvent when mixing in laboratory. The rubber formula and various additives are shown in Table 1.

*Corresponding author's e-mail: huwentaogood@163.com 
Table 1: rubber formula and parameters of various additives

\begin{tabular}{lll}
\hline Ingredients & Mass(g) & Density(g/cm3) \\
\hline Natural Latex(NL) & 120 & 0.94 \\
Znic Oxide (ZO) & 5 & 5.57 \\
Sulfur & 3 & 2.05 \\
Stearic Acid & 2 & 0.92 \\
Zinc diethyl & 1 & 1.48 \\
dithiocarbamate & & \\
Antioxidant (RD) & 1 & 1.08 \\
Potassium ethyl & 1 & 1.56 \\
xanthogenate & & \\
Graphene & 10 & 2.20 \\
\hline
\end{tabular}

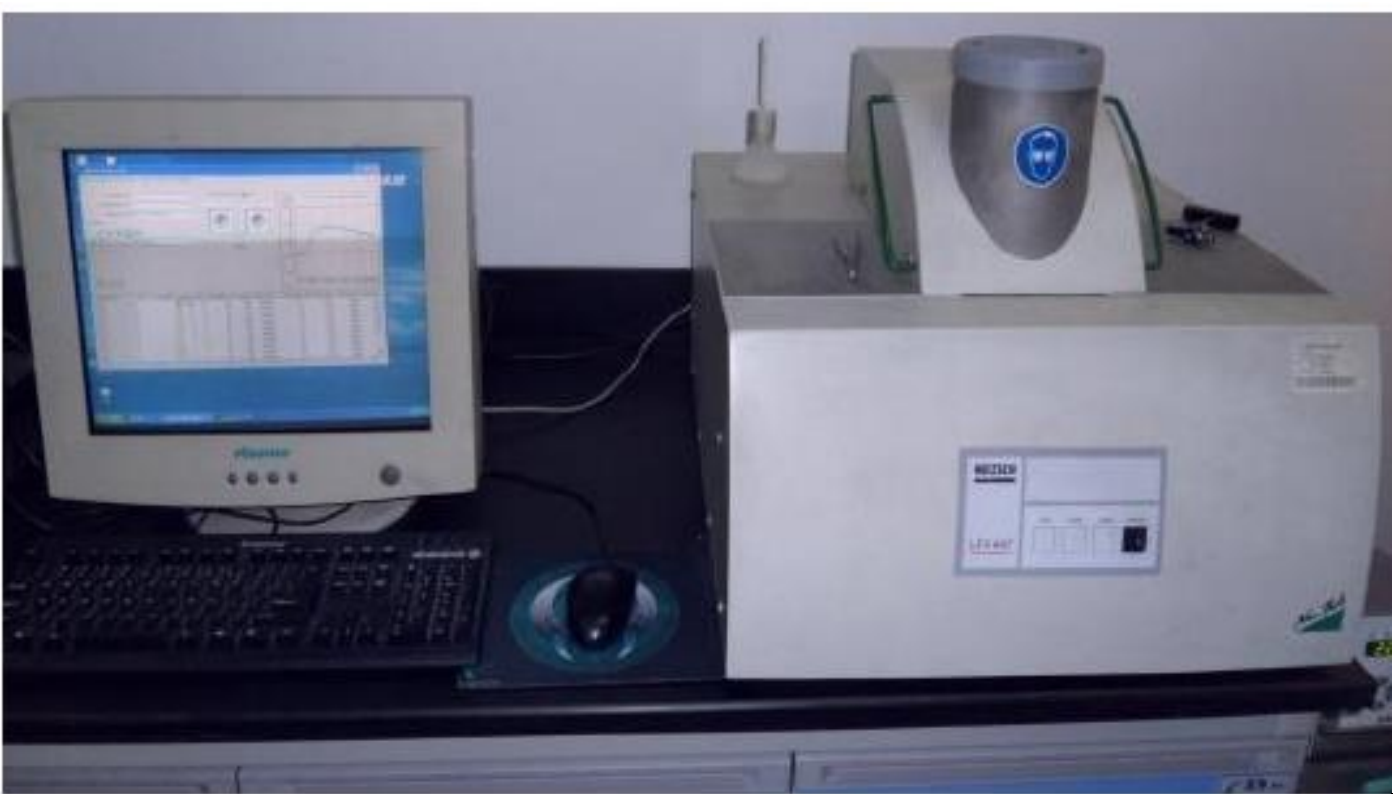

Figure 1: schematic diagram of FLA laser heat conduction instrument

\section{3 test steps}

(1) add $20 \mathrm{~g}$ natural latex to $500 \mathrm{ml}$ toluene for dissolution, add the solution to $1 \mathrm{~L}$ three port flask and stir for $1 \mathrm{~h}$.

(2) other fillers, except graphene and sulfur, were mixed into $50 \mathrm{ml}$ toluene and treated with ultrasonic for $0.5 \mathrm{~h}$ to make the dissolution complete and uniform. The amount of zinc oxide is $0.5 \mathrm{~g}$ each time, the amount of Zinc Diethyl Dithiocarbamate is $0.5 \mathrm{~g}$, the amount of stearic acid is $1 \mathrm{~g}$, the amount of antioxidant RD is $0.5 \mathrm{~g}$, and the amount of xanthic acid is $0.5 \mathrm{~g}$. Add their toluene solution to the natural rubber solution.

(3) weigh different doses of graphene and $0.3 \mathrm{~g}$ of sulfur into $50 \mathrm{ml}$ of solution, add them into natural rubber toluene solution after ultrasonic dispersion, stir for $2 \mathrm{~h}$, then stop stirring and take them out. The dosage of graphene is $0 \mathrm{~g}, 0.5 \mathrm{~g}, 1.0 \mathrm{~g}, 1.5 \mathrm{~g}, 2.0 \mathrm{~g}$ and $2.5 \mathrm{G}$ respectively. Six colloidal materials can be obtained.

(4) pour the obtained gelatinous material into the culture dish, and put it into the vacuum drying oven for vacuuming, and then put it into the oven for solvent evaporation at $30{ }^{\circ} \mathrm{C}$. When the toluene solvent is evaporated, six rubber films are formed, with a thickness

\section{2 experimental instruments}

Equipment used in the experiment: fume hood, iron stand, mixer, three port flask, glass rod, balance, measuring cylinder, etc. Fla laser thermal conductivity meter used for fla447 thermal conductivity measurement is produced by German delay resistant company, as shown in Figure 1. Its principle is to irradiate a laser beam on the sample and test the temperature change of the sample within a certain period of time, so as to obtain the curve of temperature change with time in the process of temperature rising and cooling of the sample, fit the thermal diffusivity of the thermal conductive material according to the curve, and deduce the thermal conductivity of the material from the thermal diffusivity of the thermal conductive material. of about $0.5 \mathrm{~mm}$, which are type I, type II, type III, type $\mathrm{IV}$, type $\mathrm{V}$ and type VI respectively.

(5) put 6 samples into the FLA laser thermal conductivity meter, set the temperature to $30{ }^{\circ} \mathrm{C}$, and measure the thermal conductivity of the samples.

\section{Results and discussion}

As shown in Figure 2, since graphene is not added to type I, the thermal conductivity is very low, less than $0.2 \mathrm{~W} /(\mathrm{m} \mathrm{K})$. When $0.5 \mathrm{~g}$ and $1 \mathrm{~g}$ graphene are added, the thermal conductivity of type II and type III is improved. Compared with type I without graphene, the thermal conductivity of type II and type III is increased by $30 \%-40 \%$. When the dosage of graphene continues to increase to $1.5 \mathrm{~g}$, the thermal conductivity of the composite increases rapidly, exceeding $0.6 \mathrm{~W} /(\mathrm{m} \mathrm{K})$. Compared with the type I without graphene, the thermal conductivity of type IV increases 3-4 times. With the increase of the dosage of graphene, the thermal conductivity of the composite sample also increases gradually. The reason is that the thermal conductive filler gradually replaces the matrix and becomes the main body 
of the heat conduction. At this time, the speed of the increase of the thermal conductivity is obviously accelerated.

When the dosage of graphene continues to increase to $2 \mathrm{G}$ and $2.5 \mathrm{~g}$, that is to say, the ratio of graphene dosage to natural latex is about $1: 10$. Although the thermal conductivity of type V and type VI has been improved, it is not too significant. The reason is that when the heat conduction network chain of the heat conduction filler is gradually formed, the influence of the increase of graphene dosage on its heat conduction performance is gradually reduced, so the increase speed of the heat conduction coefficient is reduced.

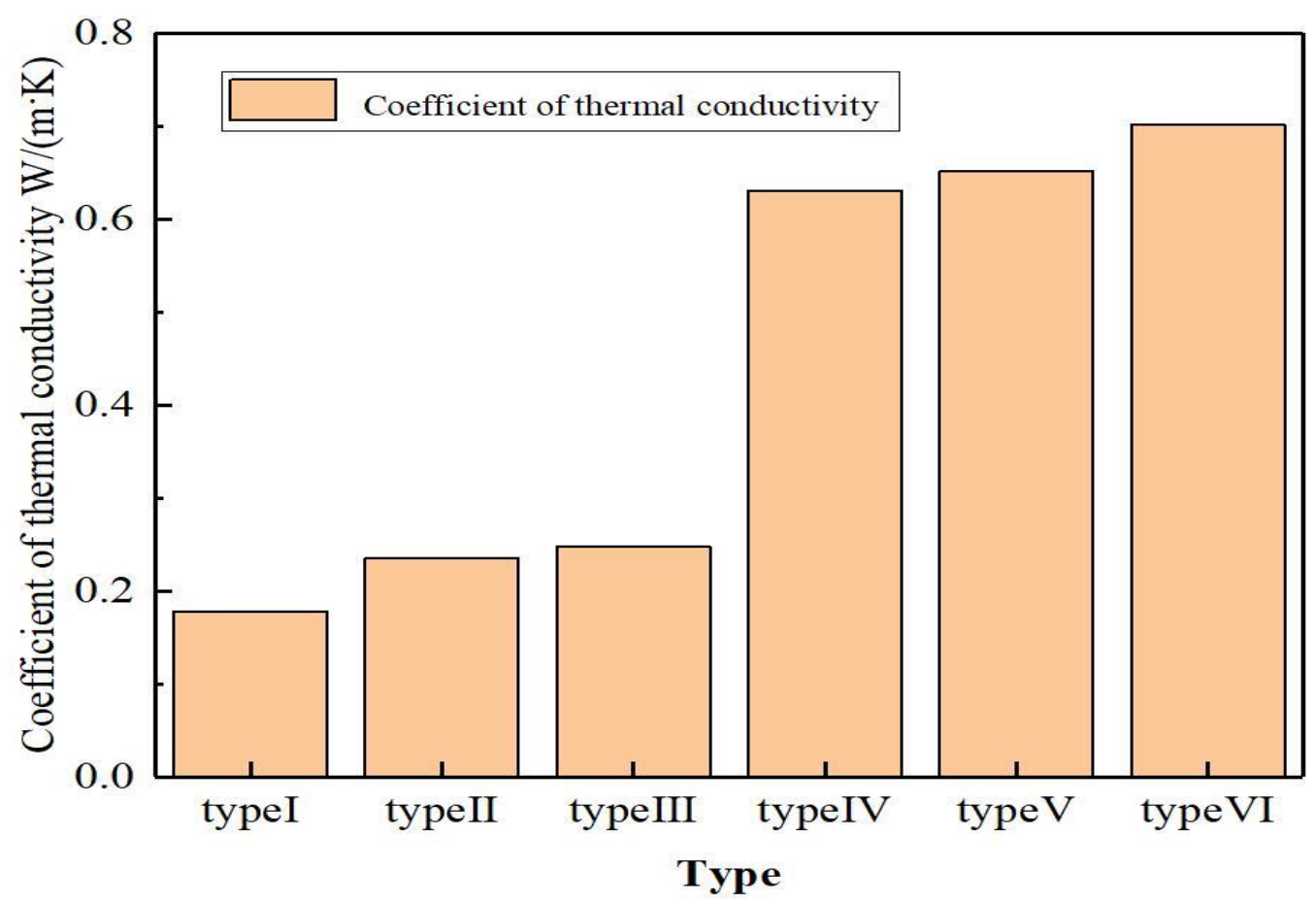

Figure 2: schematic diagram of thermal conductivity of 6 samples

\section{4 conclusion}

In order to study the influence of the blending ratio of graphene and rubber on the thermal conductivity, the composite samples with different blending ratio of graphene and rubber were tested in this experiment. The conclusions are as follows:

(1) From the numerical value of the thermal conductivity, the thermal conductivity of graphene to the natural latex is significantly improved, and the thermal conductivity of the composite sample increases with the increase of graphene dosage.

(2) With the increase of graphene dosage, when the ratio of graphene dosage to natural latex is about 1:10, the increasing speed of graphene to natural latex thermal conductivity decreases.

\section{Reference}

1. Du. M, et al. (2015) Carboxylated butadiene-styrene rubber/halloysite nanotube nanocomposites: interfacial interaction and performance [J]. Polymer,49(22):4871876.

2. Chen. W, et al. (2011) Interfacial structure and performance of rubber/boehmite nanocomposites modified by methacrylic acid [J]. Polymer, 52 (19): 4387-4395.
3. Kim. Y. A, Hayashi T, Endo M, et al. (2016) Fabrication of aligned carbon nanotube-filled rubber composite [J]. Scripta Materialia, 54 (1): 31-35.

4. Dai J C, Huang J T. (2011) Surface modification of clays and clay-rubber composite [J]. Applied clay science, 15 (1): 51-65.

5. Nethravathi C, Rajamathi .M, )2012) Chemically modified graphene sheets produced by the solvothermal reduction of colloidal dispersions of graphite oxide[J] . Carbon.46(14): 1994 1998.

6. Wang G, Shen X, Yao J,et a1(2014). Graphene nanosheets for enhanced lithium storage in lithium ion batteries[J] . Carbon. (47) 2049 2053.

7. Disma, et a1. (2016) Effect of mechanical grinding on the lithium intercalation process in graphites and soft carbons[J] Electrochem. Soc, 143(1 2): 3959 3972.

8. Sawai K, Ohzuku. (2012) Factors affecting rate capability of graphite electrodes for lithium-ionbatteries [J] Electrochem. 2013,150: A674 A678.

9. Longun J, Iroh J O. (2012) Nano-graphene/polyimide composites with extremely highrubbery plateau modulus. Carbon, 
50(5): 1823-1832

10. Xu Y F, Wang Y, Liang J, et al., (2004) A Hybrid Material of Graphene and Poly[3,4-ethyldioxythiophene] with High Conductivity, Flexibility, and Transparency. NanoResearch., 20092(4): 343-348.

11. Qu L, Huang G, Zhang P, et al. (2010) Synergistic reinforcement of nanoclay and carbon black in natural rubber $[\mathrm{J}]$. Polymer International, 59 (10): 1397-1402.

12. Chen G, Weng W, Wu D, et al. (2004) Preparation and characterization of graphite nanosheets from ultrasonic powdering technique [J]. Carbon, 42 (4): 753-759. 